\title{
Change of Receptor Binding Preference of Novel Avian Origin H7N9 in China from 2013 to 2014
}

\author{
Wei Hu \\ Department of Computer Science, Houghton College, Houghton, USA \\ Email: wei.hu@houghton.edu \\ Received 10 January 2015; accepted 28 January 2015; published 2 February 2015 \\ Copyright (C) 2015 by author and Scientific Research Publishing Inc. \\ This work is licensed under the Creative Commons Attribution International License (CC BY). \\ http://creativecommons.org/licenses/by/4.0/ \\ (c) (i) Open Access
}

\section{Abstract}

The advent of a novel avian origin H7N9 emerged in China in March 2013 is of major health concern, as it has little virulence in birds but can cause severe illness and death in humans. As people continue to get infected by this new virus in 2014 , we need to understand the current status and trend of its evolution at the end of year 2014. The influenza viruses have two surface proteins, haemagglutinin (HA) and neuraminidase (NA), which are involved in viral entry into and egress from the host cells respectively. In a previous study published in May 2013, we found that the HA protein of this new human H7N9 virus was able to preferentially bind to the avian type receptors as its primary binding and human type receptors as its secondary, which was confirmed by several web lab experiments a few months later in 2013. The current study examined the binding preference of human H7N9 again trying to uncover any change in the HA binding properties as this virus ran its course from 2013 to 2014 . Our analysis concluded that the HA binding patterns of this novel virus were stable and similar to avian H7N9 in Asia and in Europe until August 2013, but after that time the virus gradually started to change and exhibit enhanced binding features of avian H7N9 in North America. Further investigation of the observed change identified a few positions in HA that could be potentially important for distinguishing the HA sequences of human H7N9 in China collected before and after August 2013. As a result, we discovered a single amino acid substitution R47K in HA that was believed to be the primary cause for this shift of binding patterns. Finally, our findings also implied that the human infections with H7N9 in China in 2013 were more likely caused by chickens than by ducks.

\section{Keywords}

H7N9, Influenza, Receptor Binding Specificity, HA Gene 


\section{Introduction}

The emergence of new influenza viruses is a constant public health concern. In March 2013, a novel avian origin H7N9 virus was discovered in China that can infect humans and cause death. This novel H7N9 strain is the first H7 subtype discovered in Asia that is transmittable from birds to humans. A typical pathway of such infection is from migratory birds to domestic ducks, to chickens, then possibly to humans. In general, however, avian H7N9 is considered as low pathogenic, which makes it difficult for detection and control as birds infected by this virus may show no symptom at all. The World Health Organization updated as of 21 November 2014 that from 14 to 20 November 2014, 3 new cases of human H7H9 infection were reported from China in the western pacific region [1]. The fact that people in China continue to get infected by this new virus raises concerns about its ability to transmit in the human population and reminds us of the urgent need to know what is happening inside this virus today.

The eight gene segments of this new human H7N9 virus come from different avianinfluenza viruses through a sequence of events that could have involved mixing between wild and domestic ducks and reassortment in poultry. Two of the glycoproteins comprising the exterior structure of influenza viruses are haemagglutinin (HA) and neuraminidase (NA). Further, the HA protein has two subunits: HA1 subunit (globular head domain) and HA2 subunit (stem domain).

The HA protein is involved in the initial viral attachment and binding to a host cell. After infection the virus uses the host cell's reproduction machinery to multiply and eventually exit the cell through the help of NA protein which results in death of the host cell. The functions of these two proteins are complementary, i.e., the HA protein is to bind receptors on a host cell whereas NA is to destroy the receptors to enable progeny escape from the infected cell. Thus, a balance between HA and NA needs to be maintained. Moreover, an overactive NA activity could be detrimental to virus survival by destroying receptors on uninfected cells necessary for HA to bind.

The receptor binding affinity of a virus can determine what host can be infected because the first step of influenza infection is HA binding to receptors found on the host cell surface. Receptor binding properties are also critical for pathogenicity. One of the biological traits required for avian viruses to cross the species barrier to infect humans is the change of their HA binding preference. Avian and human viruses typically have a different receptor binding preference and only few amino acid changes in the HA protein could cause a switch from avian to human receptor specificity. This difference in receptor binding accounts for the reduced ability of avian strains to establish infections in humans.

The HA gene of human H7N9 is related to avian H7N3 viruses circulating in China prior to 2013, while the NA protein is similar to that of avian H11N9 and H2N9 from migratory birds, both of which are derived from the Eurasian lineage of avian viruses [2] [3]. We evaluated the HA binding of this new virus right after its advent in China in March 2013. Our findings published in May 2013 [4] suggested that the primary binding avidity of this virus is to avian types of receptors and its secondary binding is to human types, which were later verified by several wet lab experiments using glycan arrays, virus histochemistry, animal models, and structural analyses of HA [5]-[11].

The interesting part of the work in [7] is that they noticed a subtle difference in HA binding studies using a whole virus or just the HA protein alone, as the HA binding is influenced by the NA protein, which preferentially cleaves avian type receptors. Having dual binding to human and avian type receptors makes the new H7N9 virus different from avian H5N1, which bounds more strongly to avian receptors. This may be one reason why this virus is able to cause so many human infections quickly.

The purpose of the current study was to apply the methodology developed in [4] [12] [13] to examine any change in the HA binding of human H7N9 in China when it ran its course of evolution and adaptation in human population from 2013 to 2014.

\section{Materials and Methods}

\subsection{Sequence Data}

We downloaded the HA protein sequences of human and avian H7N9 in China and other regions from the Global Initiative on Sharing Avian Influenza Data (GISAID) database (http://platform.gisaid.org). 


\subsection{Informational Spectrum Method}

The informational spectrum method (ISM) is a bioinformatics approach to analyze protein sequences [3] [4] [12]. The main idea of this method is to apply Discrete Fourier Transform (DFT) to protein sequences that are represented as letters for amino acids. Therefore, before DFT can be used the protein sequences have to be transformed into numerical sequences based on electron-ion interaction potential (EIIP) of each amino acid. Then the resulting DFT coefficients are employed to produce the energy density spectrum. The informational spectrum (IS) comprises the frequencies and the amplitudes of this energy density spectrum. According to the ISM theory, the peak frequencies of IS of a protein sequence reflect its biological or biochemical functions. The ISM was successfully applied to quantify the effects of HA mutations on the receptor binding preference in [14] [16].

In [14] [15] ISM was applied to study the novel H7N9 of avian origin in China in 2013, which discovered that the primary IS frequency is $F(0.285)$ for avian receptor binding and the secondary is $F(0.326)$ for human receptor binding. We also demonstrated that human H7N9 and avian H7N9 in China in 2013 share the same HA receptor binding properties. Furthermore, mutations that could alter the HA binding preference of this new virus were also found and analyzed.

\section{Results}

The present study aimed to investigate the possible change in HA binding affinity of human H7N9 as it ran its course from 2013 to 2014. In order to shed light on any change in the receptor binding specificity of human H7N9 in China from 2013 to 2014, we compared this virus with its avian counterpart: avian H7N9 in Asia (chicken and duck), avian H7N9 in Europe, and in North America. First, the IS amplitudes at top four frequencies of human H7N9 in China were plotted in order of their collection dates (Figure 1), though not every sequence had a collection date or even a month in our whole dataset.

Our ISM analysis clearly indicated that these four IS amplitudes of human H7N9 in China remained stable until August 2013, after which they started to oscillate. The third and fourth IS amplitudes were increasing after August 2013 with the third increased more than the fourth. To investigate the meaning of this frequency $\mathrm{F}(0.166)$, we included HAs from avian H7N9 from different continents. It turned out that $\mathrm{F}(0.166)$ was the primary IS frequency for avian H7N9 in North America. For chicken HA sequences in Asia, we only had those from China, with none from other Asian countries. Unfortunately we did not have the chicken HA sequences in China after July 1 2013. Otherwise it would be interesting to see any HA binding changes in chicken population after this time.

It is commonly believed that a typical infection pathway of avian influenza viruses is from wild aquatic birds to domestic ducks, to chickens, and then maybe finally to humans. We analyzed the HAs of avian H7N9 in Asia grouped by duck and chicken, which demonstrated that the HA bindings of chicken H7N9 in Asia were closer to human H7N9 in China emerged in 2013 than to duck H7N9 in Asia (Figure 1). This also implied that the source of human infections with H7N9 in China in 2013 was more likely from chickens than from ducks. Further, the HA binding properties of avian H7N9 in Asia are closer to those in Europe than in North America (Figure 1).

To reveal any amino acid change in HA1 that might cause the observed change in HA binding of human H7N9 in China, we used Random Forest to find the important residues in HA that were critical for differentiating the HA1 sequences between before and after August 2013 (Figure 2). Next we wanted to find out, among these positions in HA1, any amino acid substitutions capable of enhancing the HA binding of human H7N9 at $\mathrm{F}(0.166)$. One consensus sequence was generated for the HA sequences before August 2013 and one for those after. Surprisingly, these two consensus sequences only differed in HA1 at residue 47 with the first consensus carrying 47R and the second 47K. The IS plots of these two consensus sequences demonstrated that the substitution R47K could increase the IS amplitude of human H7N9 at F(0.166) (Figure 3), the primary binding frequency of avian H7N9 in North America (Figure 1). Further sequence examination showed that the HA sequences of both avian H7N9 in Europe and in North America carried47R, and the first collected HA sequence containing 47K in avian H7N9 in Asia was A/wild bird/Korea/A3/11 (H7N9), suggesting the substitution R47K was a feature of H7N9 in Asia.

\section{Conclusions}

The novel human H7N9 discovered in March 2013 in China continue to infect people in 2014. Compared to 

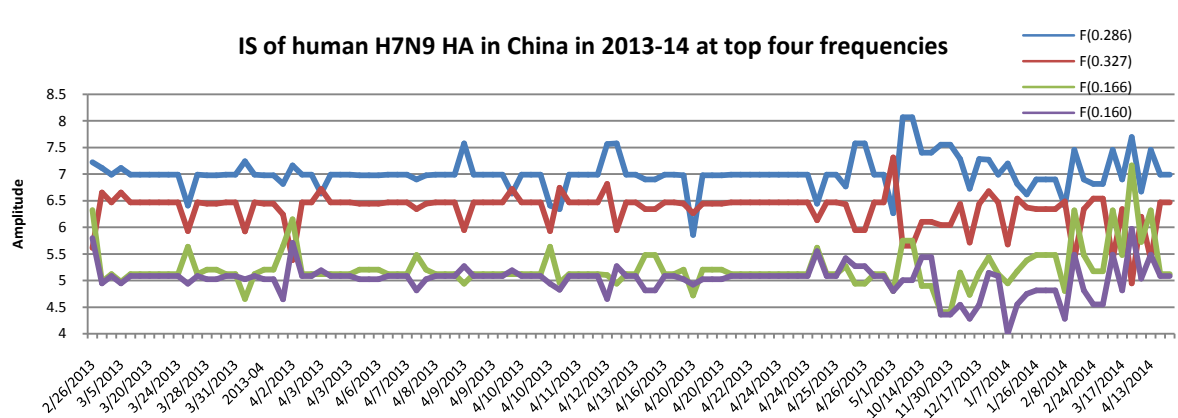

Date

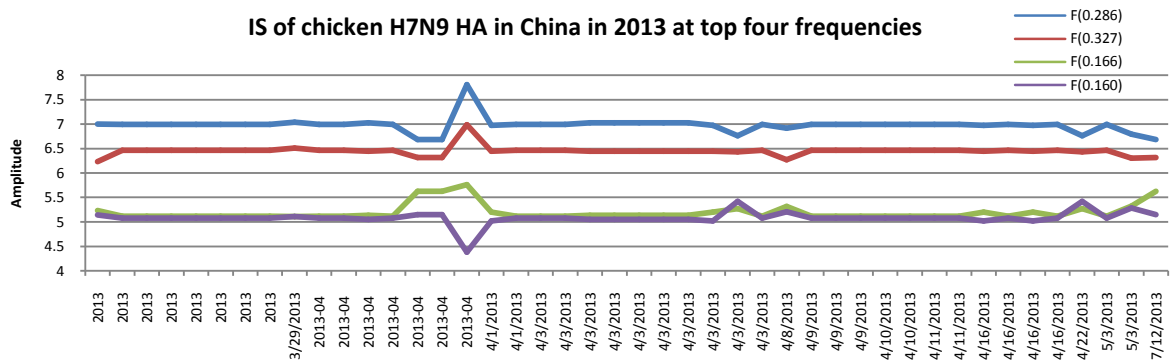

Date

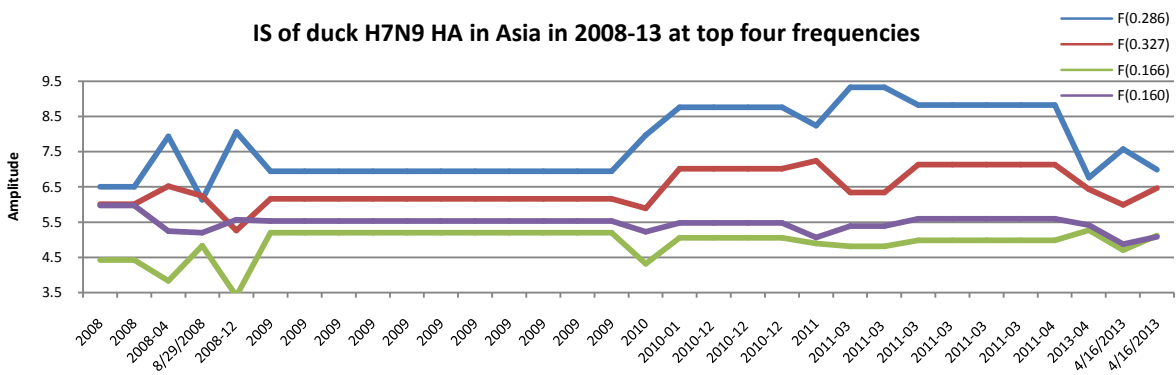

Date

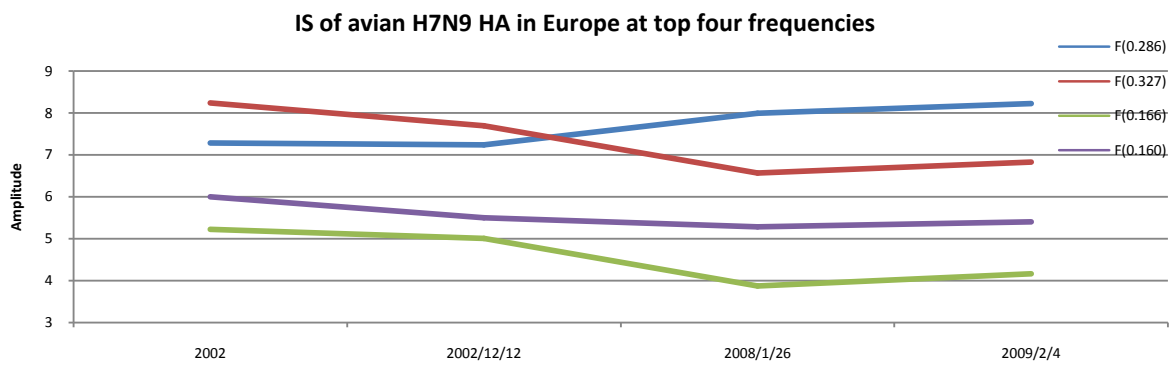

Date

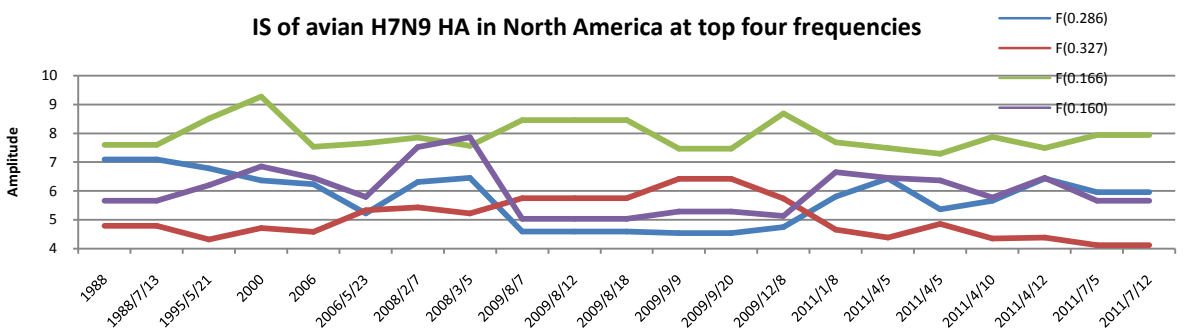

Date

Figure 1. IS of human and avian H7N9 in difference regions (All the sequences were ordered by collection dates. However, not all the sequences had a collection date, so some of them only had year or year and month). 
Top importance positions in HA1 that can distingush HA sequences of human H7N9 in China collected before and after August 2013

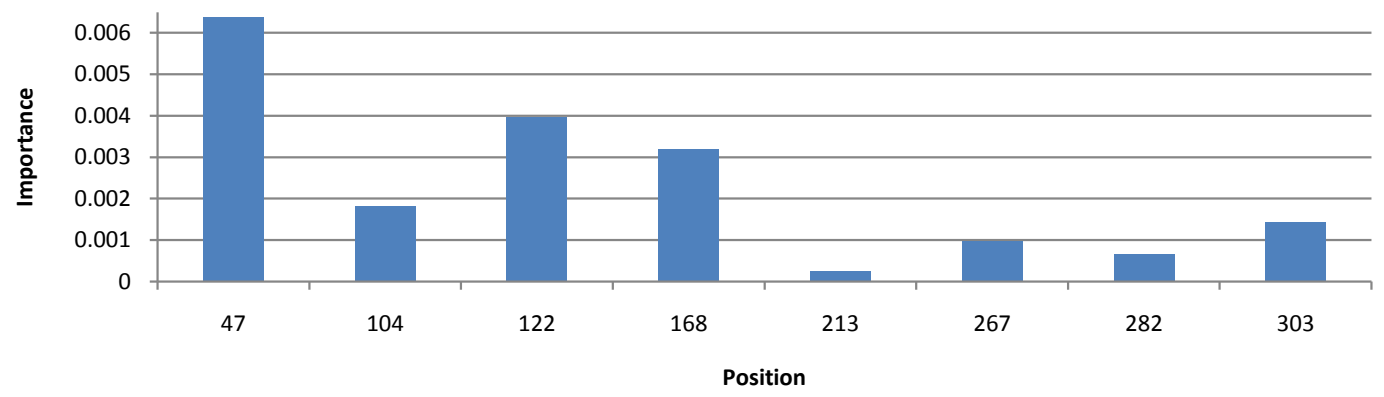

Figure 2. Top important positions in HA1 that could be used to differentiate the HA sequences of human H7N9 in China collected before and after August 2013.
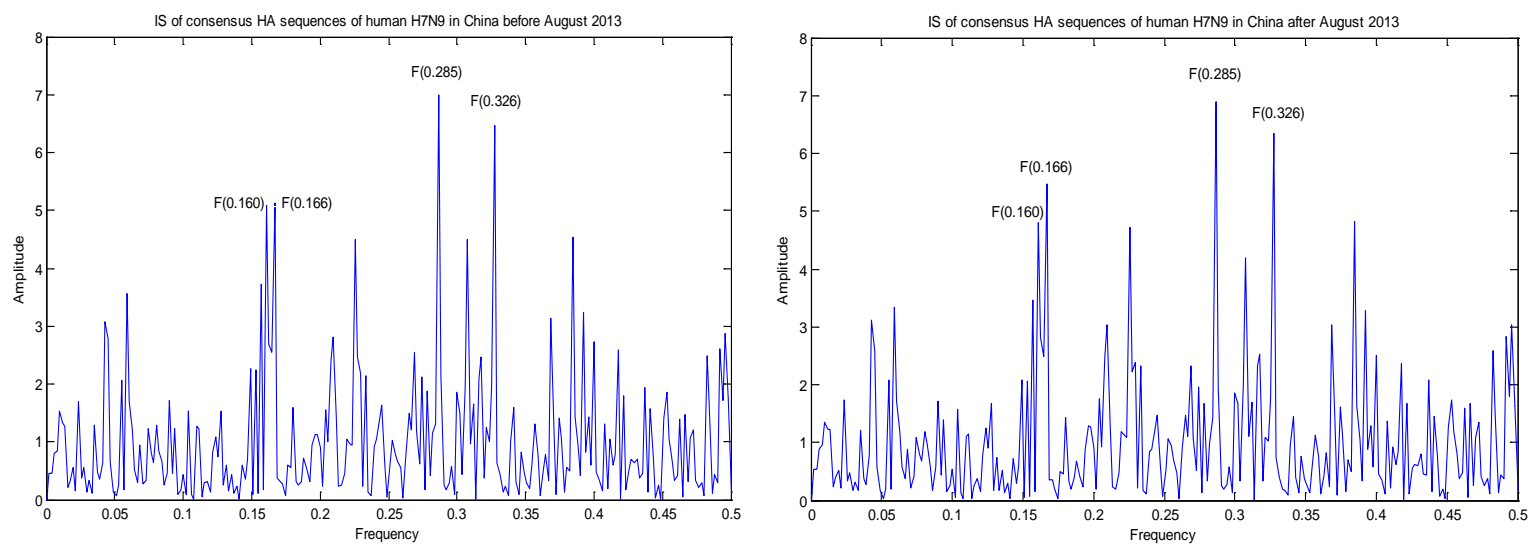

Figure 3. IS of consensus HA sequences of human H7N9 in China before or after August 2013 to show the impact of substitution R47K on the IS at $\mathrm{F}(0.166)$ as the two consensuses differed only at residue 47.

other avian influenza viruses, this H7N9 virus exhibits its ability to transmit from birds to humans more readily. This new virus shows low pathogenicity in birds but human infections can be deadly. Thus, it is of great importance to understand the extent to which the current H7N9 virus has evolved to acquire capabilities for transmission among humans. The HA protein allows influenza viruses to attach to receptors on host cells. After they get inside and replicate, the NA protein helps the new viruses to exit and find new cells to infect. Therefore, the receptor binding preference of HA is critical for viral replication and transmission.

The change of binding specificity from avian type receptors to human type receptors is thought to be a prerequisite for an avian virus to infect humans. The human H7N9 virus has dual binding to avian and human receptors, which makes it more readily to infect humans than other avian strains such as H5N1. This study took a closer look at this virus at the end of year 2014, more specifically its HA receptor binding preference. Our analysis revealed that the HA binding patterns of human H7N9 were stable and similar to its counterparts, avian H7N9 in Asian and in Europe, from March 2013 to August 2013, but after August 2013, they started to oscillate and exhibit enhanced binding patterns of avian H7N9 in North America. The uncovered change in the HA binding properties of this new virus was further explored by identification of the important resides in HA to differentiate the HA sequences of this virus collected before August 2013 and after. As a result, a single amino acid substitution R47K in HA was identified that might be responsible for this change in HA binding properties of human H7N9. We also found that the HA binding patterns of human H7N9 were closer to chicken H7N9 than to duck H7N9 in China.

\section{Acknowledgements}

We thank Houghton College for its financial support. 


\section{References}

[1] http://www.wpro.who.int/outbreaks_emergencies/H7N9/en/

[2] Neumann, G., Macken, C.A. and Kawaoka, Y. (2014) Identification of Amino Acid Changes That May Have Been Critical for the Genesis of A(H7N9) Influenza Viruses. Journal of Virology, 88, 4877-4896. http://dx.doi.org/10.1128/JVI.00107-14

[3] Liu, D., Shi, W., Shi, Y., Wang, D., Xiao, H., Li, W., Bi, Y., Wu, Y., Li, X., Yan, J., Liu, W., Zhao, G., Yang, W., Wang, Y., Ma, J., Shu, Y., Lei, F. and Gao, G.F. (2013) Origin and Diversity of Novel Avian Influenza A H7N9 Viruses Causing Human Infection: Phylogenetic, Structural, and Coalescent Analyses. Lancet, 381, 1926-1932. http://dx.doi.org/10.1016/S0140-6736(13)60938-1

[4] Hu, W. (2013) Receptor Binding Specificity and Sequence Comparison of a Novel Avian-Origin H7N9 Virus in China. Journal of Biomedical Science and Engineering, 6, 533-542. http://dx.doi.org/10.4236/jbise.2013.65068

[5] Shi, Y., Zhang, W., Wang, F., Qi, J., Wu, Y., Song, H., Gao, F., Bi, Y., Zhang, Y., Fan, Z., Qin, C., Sun, H., Liu, J., Haywood, J., Liu, W., Gong, W., Wang, D., Shu, Y., Wang, Y., Yan, J. and Gao, G.F. (2013) Structures and Receptor Binding of Hemagglutinins from Human-Infecting H7N9 Influenza Viruses. Science, 342, 243-247. http://dx.doi.org/10.1126/science.1242917

[6] Xiong, X., Martin, S.R., Haire, L.F., Wharton, S.A., Daniels, R.S., Bennett, M.S., McCauley, J.W., Collins, P.J., Walker, P.A., Skehel, J.J. and Gamblin, S.J. (2013) Receptor Binding by an H7N9 Influenza Virus from Humans. Nature, 499, 496-499. http://dx.doi.org/10.1038/nature12372

[7] Xu, R., de Vries, R.P., Zhu, X., Nycholat, C.M., McBride, R., Yu, W., Paulson, J.C. and Wilson, I.A. (2013) Preferential Recognition of Avian-Like Receptors in Human Influenza A H7N9 Viruses. Science, 342, 1230-1235. http://dx.doi.org/10.1126/science.1243761

[8] Zhu, H., Wang, D., Kelvin, D.J., Li, L., Zheng, Z., Yoon, S.W., Wong, S.S., Farooqui, A., Wang, J., Banner, D., Chen, R., Zheng, R., Zhou, J., Zhang, Y., Hong, W., Dong, W., Cai, Q., Roehrl, M.H., Huang, S.S., Kelvin, A.A., Yao, T., Zhou, B., Chen, X., Leung, G.M., Poon, L.L., Webster, R.G., Webby, R.J., Peiris, J.S., Guan, Y. and Shu, Y. (2013) Infectivity, Transmission, and Pathology of Human H7N9 Influenza in Ferrets and Pigs. Science, 341, 183-186. http://dx.doi.org/10.1126/science.1239844

[9] Watanabe, T., Kiso, M., Fukuyama, S., Nakajima, N., Imai, M., Yamada, S., Murakami, S., et al. (2013) Characterization of H7N9 Influenza A Viruses Isolated from Humans. Nature, 501, 551-555. http://dx.doi.org/10.1038/nature12392

[10] Belser, J.A., Gustin, K.M., Pearce, M.B., Maines, T.R., Zeng, H., Pappas, C., Sun, X., Carney, P.J., Villanueva, J.M., Stevens, J., Katz, J.M. and Tumpey, T.M. (2013) Pathogenesis and Transmission of Avian Influenza A (H7N9) Virus in Ferrets and Mice. Nature, 501, 556-559. http://dx.doi.org/10.1038/nature12391

[11] Ramos, I., Krammer, F., Hai, R., Aguilera, D., Bernal-Rubio, D., Steel, J., García-Sastre, A. and Fernandez-Sesma, A. (2013) H7N9 Influenza Viruses Interact Preferentially with $\alpha 2$, 3-Linked Sialic Acids and Bind Weakly to $\alpha 2$, 6Linked Sialic Acids. Journal of General Virology, 94, 2417-2423. http://dx.doi.org/10.1099/vir.0.056184-0

[12] Hu, W. (2013) Mutations in Hemagglutinin of a Novel Avian-Origin H7N9 Virus That Are Critical for Receptor Binding Specificity. Tsinghua Science and Technology, 18, 522-529. http://dx.doi.org/10.1109/TST.2013.6616525

[13] W. Hu (2014) Functional Interplay between Hemagglutinin and Neuraminidase of Pandemic 2009 H1N1 from the Perspective of Virus Evolution. Lecture Notes in Computer Science, 8492, 38-49.

[14] Cosic, I. (1997) The Resonant Recognition Model of Macromolecular Bioreactivity, Theory and Application. Birkhauser Verlag, Berlin.

[15] Veljkovic, V., Niman, H.L., Glisic, S., et al. (2009) Identification of Hemag-Glutinin Structural Domain and Polymorphisms Which May Modulate Swine H1N1 Interactions with Human Receptor. BMC Structural Biology, 9, 62. http://dx.doi.org/10.1186/1472-6807-9-62

[16] Veljkovic, V., Veljkovic, N., Muller, C.P., et al. (2009) Characterization of Conserved Properties of Hemagglutinin of H5N1 and Human Influenza Viruses: Possible Consequences for Therapy and Infection Control. BMC Structural Biology, 7, 9-21. 
Scientific Research Publishing (SCIRP) is one of the largest Open Access journal publishers. It is currently publishing more than 200 open access, online, peer-reviewed journals covering a wide range of academic disciplines. SCIRP serves the worldwide academic communities and contributes to the progress and application of science with its publication.

Other selected journals from SCIRP are listed as below. Submit your manuscript to us via either submit@scirp.org or Online Submission Portal.
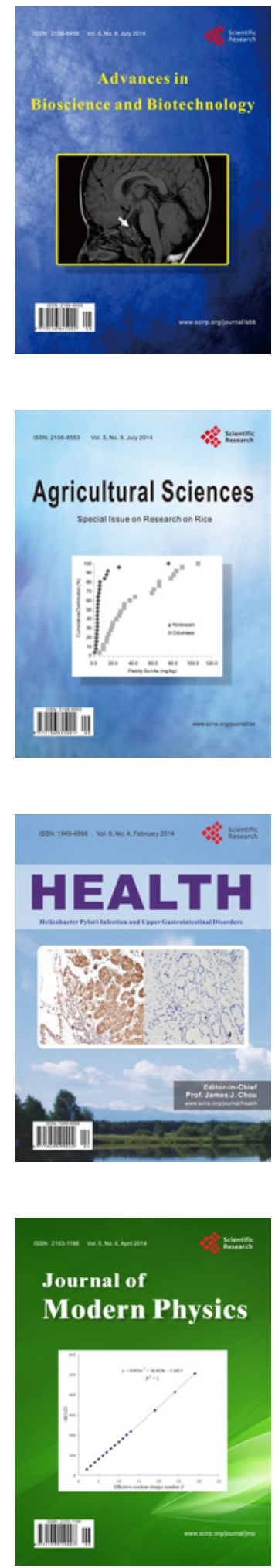
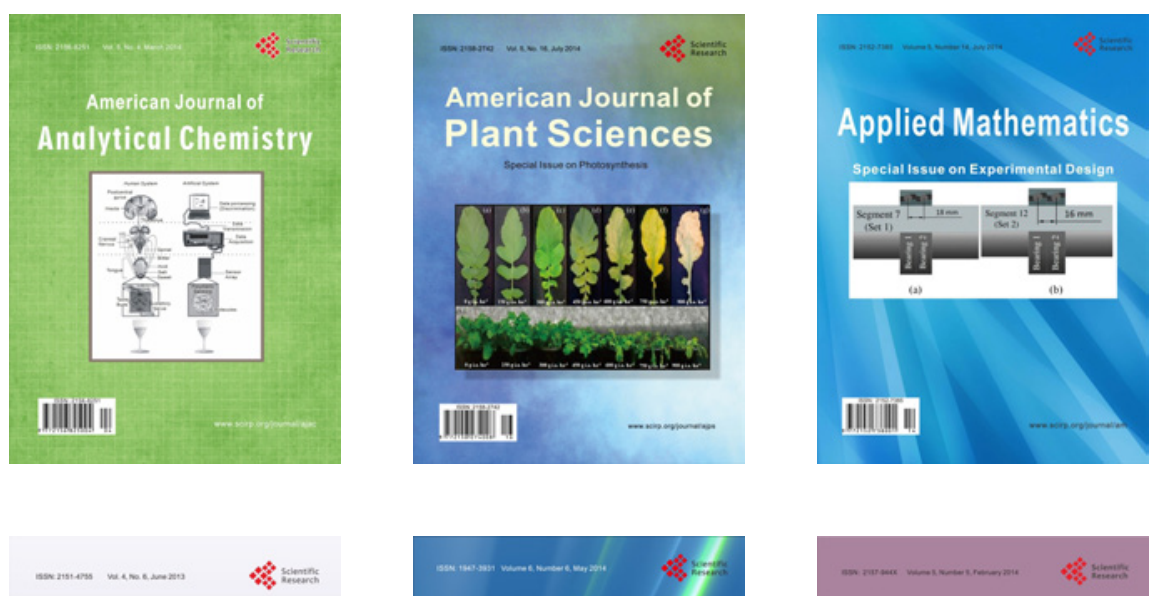

Creative Education
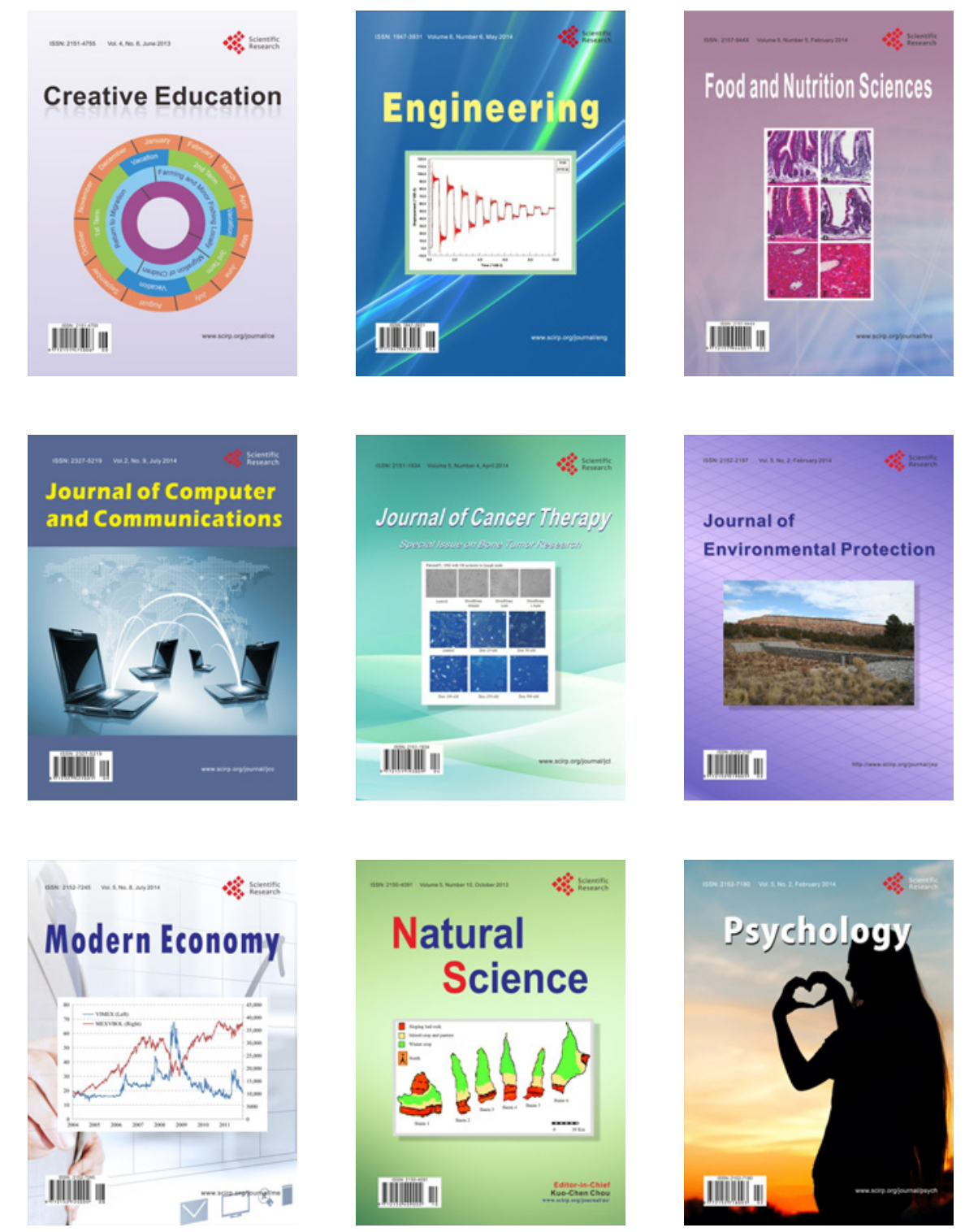